\title{
Measuring the Overall Security of Network Configurations Using Attack Graphs
}

\author{
Lingyu Wang ${ }^{1}$, Anoop Singhal ${ }^{2}$, and Sushil Jajodia ${ }^{3}$ \\ ${ }^{1}$ Concordia Institute for Information Systems Engineering \\ Concordia University \\ Montreal, QC H3G 1M8, Canada \\ wang@ciise.concordia.ca \\ ${ }^{2}$ Computer Security Division, NIST \\ Gaithersburg, MD 20899, USA \\ anoop.singhalenist.gov \\ ${ }^{3}$ Center for Secure Information Systems \\ George Mason University \\ Fairfax, VA 22030-4444, USA \\ jajodia@gmu.edu
}

\begin{abstract}
Today's computer systems face sophisticated intrusions during which multiple vulnerabilities can be combined for reaching an attack goal. The overall security of a network system cannot simply be determined based on the number of vulnerabilities. To quantitatively assess the security of networked systems, one must first understand which and how vulnerabilities can be combined for an attack. Such an understanding becomes possible with recent advances in modeling the composition of vulnerabilities as attack graphs. Based on our experiences with attack graph analysis, we explore different concepts and issues on a metric to quantify potential attacks. To accomplish this, we present an attack resistance metric for assessing and comparing the security of different network configurations. This paper describes the metric at an abstract level as two composition operators with features for expressing additional constraints. We consider two concrete cases. The first case assumes the domain of attack resistance to be real number and the second case represents resistances as a set of initial security conditions. We show that the proposed metric satisfies desired properties and that it adheres to common sense. At the same time, it generalizes a previously proposed metric that is also based on attack graphs. It is our belief that the proposed metric will lead to novel quantitative approaches to vulnerability analysis, network hardening, and attack responses.
\end{abstract}

\section{Introduction}

Today's networked computer systems constitute the core component of information technology infrastructures in enterprises and in critical infrastructures, such as power grids, financial data systems, and emergency communication systems. Protecting such systems against malicious intrusions is crucial to the economy and to our national security. Having a standard way for measuring various aspects of network security will bring together users, vendors, and labs in specifying, implementing, and evaluating the requirements and features of network security products. However, in spite of various

S. Barker, G.-J. Ahn (Eds.): Data and Applications Security 2007, LNCS 4602, pp. 98 112 2007.

(C) IFIP International Federation for Information Processing 2007 
efforts in standardizing security metric, a widely-accepted metric for network security is still largely unavailable. This is partly due to the fact that most researchers are still adopting a qualitative and imprecise view toward the evaluation of network security. For example, typical issues addressed in current research may ask following questions. Are all critical resources in a network secure (topological vulnerability analysis)? Can a network be hardened to secure the given resources (network hardening)? How to stop an ongoing intrusion from compromising given resources (attack response)?

The qualitative nature of these questions reflect the current focus on the qualitative, rather than quantitative, study of network security. This focus implies the inherent impreciseness in many research results and also indicates the need for more research efforts on security metrics. However, the lack of research on quantitative aspects of network security is natural. Assessing the overall security of a network requires a thorough understanding of the interplay between host vulnerabilities. That is, which and how vulnerabilities can be combined for an attack. Such an understanding is difficult to obtain with existing security tools, such as vulnerability scanners and intrusion detection systems. These tools typically focus on identifying individual vulnerabilities or attacks, and are usually unaware of the relationships among vulnerabilities or attacks.

Recent advances in modeling compositions of vulnerabilities using attack graphs (a review of related work will be given in the next section) indicate that the research has progressed to a point where the quantitative study of network security is critical and, at the same time, possible. Attack graphs supplement vulnerability scanners with the missing information about relationships among vulnerabilities. Analyzing the correlated vulnerabilities thus provides a clear picture about what attacks might happen in a network and about their consequences. Attack graphs thus allow us to consider potential attacks in a particular context relevant to the given network. The current work is based on our past experiences with attack graph analysis [12 |15|16 21|29|30|31|32] and a practical tool, the Topological Vulnerability Analysis (TVA) system, with the capability of modeling more than 37,000 vulnerabilities taken from 24 information sources including X-Force, Bugtraq, CVE, CERT, Nessus, and Snort [12]. The presence of such a powerful tool demonstrates the practicality of using attack graphs as the basis for measuring network security.

Instead of measuring individual vulnerabilities and then wondering about their combined effect, this paper measures the overall security of a network using the context provided by an attack graph. Such a capability will enable us to answer important questions like (but not limited to): How much effort and time will it take to compromise a critical resource under each possible network configuration? Answers to such questions will allow system administrators to choose the optimal configuration that is the most resistant to potential attacks. More specifically, we propose an attack resistance metric for assessing and comparing the security of different network configurations. The metric is based on intuitive properties derived from common sense. For example, our metric will indicate reduced security when more attack paths exist, whereas it indicates increased security for longer and more difficult paths. To make the metric broadly applicable, we first describe it at an abstract level as two composition operators with functions that allow for expressing additional dependency relationship between resistances. We then consider two concrete cases. The first assumes the domain of attack resistance to be 
real number and the second represents resistances as sets of initial security conditions. For the first case, we propose to use operators that are analogous to the ones used in computing the resistance of a series and parallel circuit. We study additional issues that arise due to the unique properties of attack graphs. For the second case, we show that a previously proposed metric [21] is equivalent to our metric under certain conditions.

The rest of the paper is organized as follows. Section 2 outlines a framework for defining security metrics using attack graphs. Section 3 presents the attack resistance metric. Section 4 reviews related work. Finally, Section 5 concludes the paper.

\section{A Framework for Defining Security Metrics Using Attack Graphs}

This section first reviews the attack graph model and then discusses intuitions behind the proposed metric.

\subsection{Attack Graph Model}

We adopt the attack graph model used in the Topological Vulnerability Analysis tool [12], which is one of the most advanced utilities for generating and analyzing attack graphs. This attack graph model is similar in nature to the earlier ones based on modified model checking [26], but it avoids the potential combinatorial explosion faced by the latter. More specifically, it makes a monotonicity assumption stating an attacker never relinquishes an obtained capability [1]. An attack graph can thus record the dependency relationship between exploits instead of recording all attack paths. The resulting attack graph has no duplicate vertices and hence has a polynomial size in the number of vulnerabilities multiplied by the number of connected pairs of hosts.

In our model, an Attack graph is a directed graph representing prior knowledge about vulnerabilities, their dependencies, and network connectivity. The vertices of an attack graph are divided into two categories, namely, exploits and security conditions (or simply conditions when no confusion is possible). First, exploits are actions taken by attackers on one or more hosts in order to take advantage of existing vulnerabilities. We denote an exploit as a predicate. For example, an exploit involving three hosts can be denoted using $v\left(h_{s}, h_{m}, h_{d}\right)$, which indicates an exploitation of the vulnerability $v$ on the destination host $h_{d}$, initiated from the source host $h_{s}$, through an intermediate host $h_{m}$. Similarly, we write $v\left(h_{s}, h_{d}\right)$ or $v(h)$, respectively, for exploits involving two hosts (no intermediate host) or one (local) host.

Second, a security condition is a property of the system or network that is relevant to some exploits. A condition is relevant to an exploit if it is either required for executing the exploit or satisfied by executing the exploit. We also use a predicate to represent a condition involving one or more hosts. For example, $c\left(h_{s}, h_{d}\right)$ indicates a securityrelated condition $c$ involving the source host $h_{s}$ and the destination host $h_{d}$. Similarly, a condition that only involves a single host can be written as $c(h)$. Examples of security conditions include the existence of a vulnerability, the existence of network connectivity or trust relationship between two hosts. It is worth noting that an attack graph usually includes exploits and conditions corresponding to normal services or functionality. Such services are included because they may help attackers in escalating their 
privileges when combined with other exploits, although they are not intended for that purpose. On the other hand, this fact also implies that not all exploits can be removed in hardening a network, so measuring the relative security of different configurations becomes important.

Directed edges in an attack graph inter-connect exploits with conditions. No edge directly goes between two exploits or between two conditions. First, an edge from a condition to an exploit denotes the require relation, which means the exploit cannot be executed unless the condition is satisfied. Second, an edge pointing from an exploit to a condition denotes the imply relation, which means executing the exploit will satisfy the condition. For example, an exploit typically requires at least two conditions, that is the existence of the vulnerability (which could be a normal service) on the destination host and the network connectivity between the two hosts. We formally characterize attack graphs in Definition 1

Definition 1. Given a set of exploits $\mathcal{E}$, a set of conditions $\mathcal{C}$, and two relations require $\subseteq \mathcal{C} \times \mathcal{E}$ and imply $\subseteq \mathcal{E} \times \mathcal{C}$, an attack graph $G$ is the directed graph $G(\mathcal{E} \cup \mathcal{C}$, require $\cup$ imply $)(\mathcal{E} \cup \mathcal{C}$ is the vertex set and require $\cup$ imply the edge set $)$.

One important semantics of attack graphs is that the require relation is conjunctive, whereas the imply relation is disjunctive. More precisely, an exploit cannot be realized until all of its required conditions have been satisfied, whereas a condition is satisfied if any of the realized exploits implies that condition. Sometimes only exploits in an attack graph are of interest, we thus remove conditions to obtain an exploit dependency graph. However, in such a graph, edges between exploits may represent both the conjunctive and disjunctive relationship. For example, in an attack graph, if two exploits $e_{1}$ and $e_{2}$ both imply the same condition $c_{1}$, which is required by another exploit $e_{3}$, then $e_{3}$ can be executed after executing either $e_{1}$ or $e_{2}$ (since $c_{1}$ will be satisfied by any of them). On the other hand, if $e_{1}$ implies $c_{1}, e_{2}$ implies a different condition $c_{2}$, and both $c_{1}$ and $c_{2}$ are required by $e_{3}$, then $e_{3}$ cannot be executed before both $e_{1}$ and $e_{2}$ are. We shall need this observation later in the paper.

\subsection{Motivating Example}

To build intuitions about properties that a security metric should satisfy, we consider the well-known attack scenario as shown on the left hand side of Figure 1 (notice that this is an overly simplified example for illustration purposes, and our metric and techniques are intended for more complicated cases where results cannot be obtained through observations). In this attack graph, exploits are depicted in ovals and conditions in clear text. The critical condition that needs to be guarded is shown in a shaded oval. The attack graph basically indicates that an attacker on host 0 can obtain user privilege on host 1, either using an SSH buffer overflow attack or through the trust relationship established by uploading the .rhost file through FTP. The attacker can use the latter trick to obtain user privilege on host 2 , either directly from host 0 or using host 1 as an intermediate stepping stone. The attacking goal, that is the root privilege on host 2 , can then be obtained using a local buffer overflow attack.

The right hand side of Figure 1 shows the exploit dependency graph. It is worth noting that in this specific case only disjunctive dependency relationship exists between 


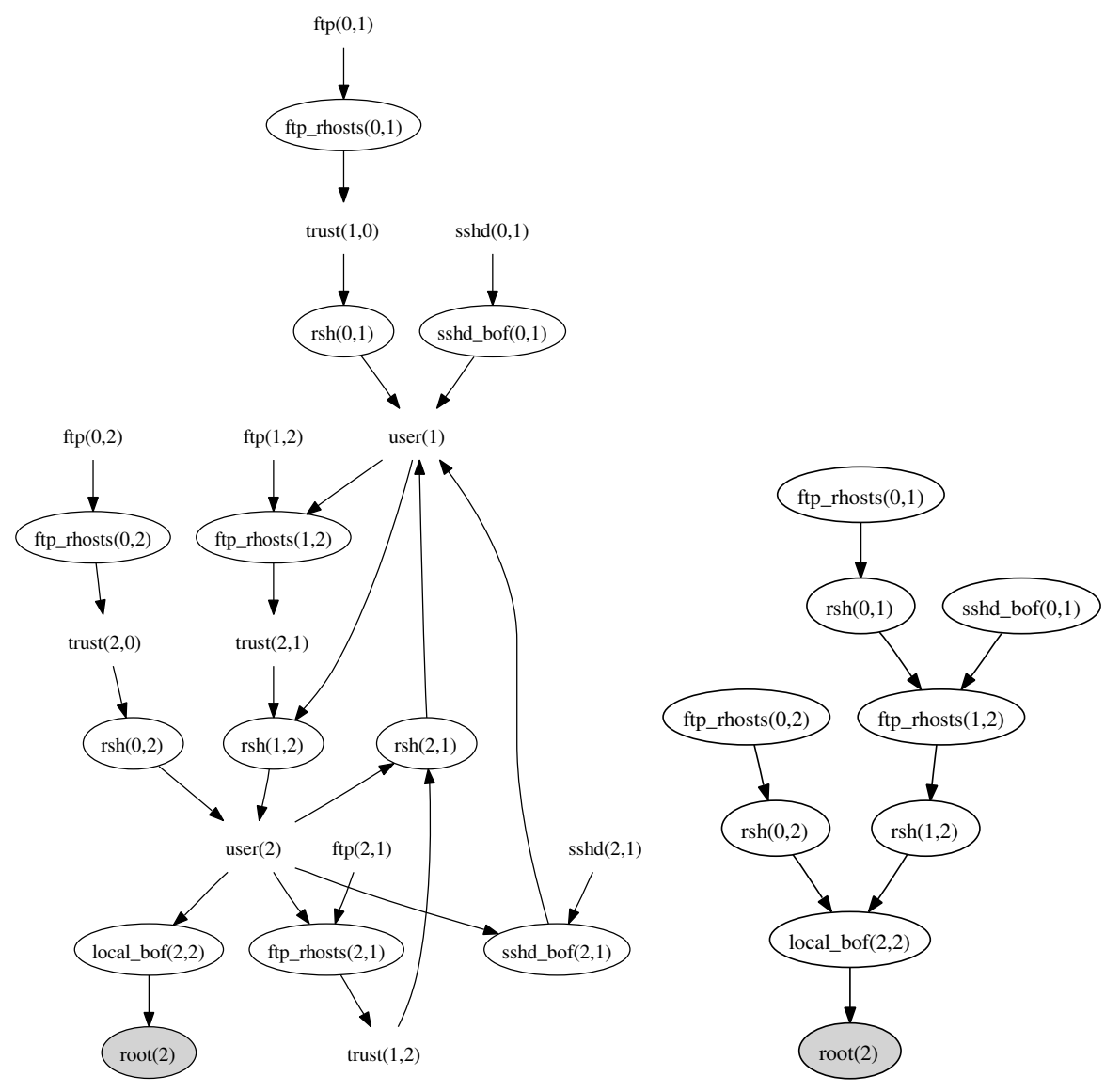

Fig. 1. An Example of Attack Graph and Exploit Dependency Graph

exploits. For example, there are two alternative ways to reach the exploit ftp_rhosts $(1,2)$ and similarly two ways to reach the exploit local_bof $(2,2)$. In general, the dependency relationship between exploits can be both disjunctive and conjunctive, and the graph notation is thus not sufficient to distinguish between the two. Later we shall introduce a special notation for this purpose.

We make several observations in Figure1, First, the two loops via exploit $f t p \_r h o s t s$ $(2,1)$ and sshd_bof $(2,1)$ are both removed. These loops both allow the attacker to obtain user privilege on host 1 for the second time, after the privilege has already been obtained (otherwise the two exploits cannot be executed). An attacker can certainly make such redundant attacking effort at will, but a security metric should assume the most efficient attackers and indicate the security of a network in the worst-case scenario. That is, a metric should never yield a value that is greater than the smallest attacking effort required for reaching the attack goal. 
Second, the exploits in Figure 1 clearly have different difficulty in terms of the time and effort required for their execution. For example, the ftp_rhosts and $r s h$ exploits both take advantage of normal services in a clever way, and they usually do not require much time or effort if the attacker has the basic knowledge about the attack (between the two type of exploits, $r s h$ may be slightly easier than ftp_rhosts in the sense that the latter requires crafting the .rhost file). On the other hand, both the sshd_bof and the local_bof are buffer overflow attacks, which require significantly more knowledge and time than the previous two because a buffer overflow attack usually requires brute force effort to determine proper parameters. This example thus shows different exploits have different difficulties in terms of effort and time required for their execution.

Third, there are three possible attack paths (that is, sequences of attacks) reaching the attack goal, as shown on the right hand side of Figure 1 the left path (that is, the one through ftp_rhosts $(0,2)$ and $r \operatorname{sh}(0,2))$ requires the smallest amount of effort. The middle path requires slightly more effort since it involves both host 1 and host 2 . The right path demands the most effort because it requires an additional buffer overflow attack sshd_bof $(0,1)$. Recall the above argument that security should be measured as the smallest effort required to reach the goal. It seems that the left path is a good candidate to be used as the measure of overall security. However, it is important to notice that when multiple paths coexist in an attack graph, reaching the attack goal is actually easier than if only one of these paths exists (even if the path requires the smallest amount of effort). Intuitively, more attack opportunities mean less security, because attackers will have a better chance to reach the attack goal. In this specific case, even though the middle and the right paths are more difficult than the left one, they nevertheless represent possibilities for attacks and thus they do reduce the overall security of the network. That is, multiple attack paths together are less secure than any of the paths alone.

Finally, assuming the middle attack path is followed by an attacker, it can be argued that the exploit $f t p \_r h o s t s(1,2)$ may be slightly easier than its predecessor ftp_rhosts $(0,1)$. To launch the same type of attack for the second time, the attacker will benefit from his/her experiences and tools that have been accumulated while launching the attack for the first time. It is, however, not possible to add an edge between these two exploits in attack graph, because the exploit $f t p \_r h o s t s(1,2)$ does not directly depend on $f t p \_r h o s t s(0,1)$ (with $r \operatorname{sh}(0,1)$ in the middle). This implies that an additional relation is needed to encode such dependency relationship between exploits, which is different from the imply or require relations already encoded in attack graphs. In another word, executing an exploit may change the difficulty of executing another exploit, even if the two do not directly depend on each other in the attack graph.

The above requirements are largely common sense that should be satisfied by a security metric. The rest of the paper proposes a security metric based on the attack graph model by taking these requirements into consideration.

\section{An Attack Resistance Metric}

This section proposes an attack resistance metric based on the attack graph model. We first discuss the metric in a generic form. We then discuss two concrete cases to illustrate 
the metric in more details. We address various issues encountered while computing the metric from a given attack graph.

\subsection{A Generic Framework}

We propose to measure the attack resistance of a network configuration as the composition of measures of individual exploits. Ideally, the resistance of each type of exploits in terms of effort and time should be represented as a total order, such as using real numbers (the next section considers how individual resistances can be combined when attack resistance is represented as a real number). Unfortunately, although clearly desired, the information and resources required by this ideal situation are limited [17]. It is, however, usually possible to estimate an approximate ordering or a partial ordering on the domain of attack resistance. We shall also consider another case where the resistance of individual exploit is simply the set of initial conditions (that is, conditions not implied by other exploits).

Different applications may define the attack resistance of individual exploits in significantly different ways. To make our metric broadly applicable, we describe the metric in a generic form while leaving the individual measures uninterpreted. Central to the model are two types of composition operators, denoted as $\oplus$ and $\otimes$. The two operators correspond to the disjunctive and conjunctive dependency relationship between exploits in an attack graph, respectively. Based on the intuitive properties mentioned in Section 2.2, the two operators should satisfy that $r_{1} \oplus r_{2}$ is no greater than $r_{1}$ or $r_{2}$, whereas $r_{1} \otimes r_{2}$ is no less than $r_{1}$ and $r_{2}$, with respect to a given ordering on the domain of attack resistance.

In addition to the two composition operators, we introduce a function $\mathcal{R}()$ that maps a set of exploits to another exploit and its resistance value. The function is intended to capture a special kind of dependency relationship between exploits. That is, executing some exploits may affect the resistance value of another exploit, even though the latter cannot be executed yet. In most cases, this effect will be to assign a lower resistance value to the affected exploit. For example, exploits involving the same vulnerability should be related together using this function such that successfully exploiting one instance of the vulnerability reduces the resistance of others due to the attacker's accumulated experiences and tools. We shall also show that this function is useful in handling the non-tree structure of attack graphs. We summarize the model in Definition 2

Definition 2. Given an attack graph $G(\mathcal{E} \cup \mathcal{C}$, require $\cup$ imply $)$ with attack goals $g \subseteq \mathcal{C}$, the attack resistance metric is composed of

- A total function $r(): \mathcal{E} \rightarrow \mathcal{D}$,

- a total function $R(): \mathcal{E} \rightarrow \mathcal{D}$,

- an operator $\oplus: \mathcal{D} \times \mathcal{D} \rightarrow \mathcal{D}$,

- an operator $\otimes: \mathcal{D} \times \mathcal{D} \rightarrow \mathcal{D}$, and

- a function $\mathcal{R}(): \mathcal{E} \rightarrow \mathcal{E} \times \mathcal{D}$.

We call the set $\mathcal{D}$ the domain of resistance, $r(e)$ the individual resistance (or simply resistance) of an exploit $e, R(e)$ the cumulative resistance of $e$. 
The main tasks in implementing this metric for a specific application is to populate the individual resistance by defining the function $r()$, to determine suitable operators $\oplus$ and $\otimes$, to capture additional dependency relationships between exploits using the function $\mathcal{R}$, and finally to decide how the cumulative resistance function $R()$ should be computed based on these information. The cumulative resistance of each attack goal then provides a quantitative measure as how likely that attack goal can be achieved, or equivalently, how vulnerable the corresponding resource is under a given network configuration.

\subsection{Attack Resistance as Real Numbers}

We now consider a concrete case where the domain of resistance $\mathcal{D}$ is the non-negative real number. Analogous to the resistance of a series and parallel circuit, we define $\oplus$ as the reciprocal of the sum of the reciprocal of individual resistance values. That is, $\frac{1}{r_{1} \oplus r_{2}}=\frac{1}{r_{1}}+\frac{1}{r_{2}}$. The operator $\otimes$ is simply addition. Recall our discussions about the relative difficulty of different type of exploits in Section 2.2. Suppose we assign the value 10 to be the resistance of each sshd_bof and local_bof, the value 2 and 1 to each ftp_rhosts and $r s h$ exploit, respectively, as depicted on the left hand side of Figure 2. The cumulative resistances can then be computed as follows, where $r()$ stands for the individual resistance and $R($ ) the cumulative resistance (we shall not consider the function $\mathcal{R}$ for the time being). The final results are shown in the right hand side of Figure 2

$$
\begin{aligned}
& -R(r s h(0,1))=r\left(f t p \_r h o s t s(0,1)\right)+r(r s h(0,1))=2+1=3 \\
& \text { - } R\left(f t p \_r h o s t s(1,2)\right)=1 /\left(1 / R(r s h(0,1))+1 / r\left(\operatorname{sshd} \_b o f(0,1)\right)\right)+ \\
& r\left(f t p \_r h o s t s(1,2)\right)=1 /(1 / 3+1 / 10)+2 \approx 4.3 \\
& -R(r \operatorname{sh}(1,2))=R(\text { ftp_rhosts }(1,2))+r(r \operatorname{sh}(1,2)) \approx 4.3+1=5.3 \\
& -R(r \operatorname{sh}(0,2))=r\left(f t p \_r h o s t s(0,2)\right)+r(r s h(0,2))=2+1=3 \\
& \text { - } R(\text { local_bof }(2,2))=1 /(1 / R(r \operatorname{sh}(0,2))+1 / R(r \operatorname{sh}(1,2)))+ \\
& r(\text { local_bof }(2,2))=1 /(1 / 3+1 / 5.3)+10 \approx 11.9
\end{aligned}
$$

According to our discussions in Section 2.2, the cumulative resistance of the whole network should be smaller than the cumulative resistance of each possible attack path. The cumulative resistance for each attack path reaching the goal can be computed by simply adding (that is, the $\otimes$ operator) individual resistance values along the path. The results for the three attack paths in Figure 2 are 13, 16, and 23, from left to right. Clearly, the accumulative resistance of the whole network, 11.9, is indeed smaller than any of the three values, satisfying the intuitive requirements given in Section 2.2. We may also notice that the composition (using the operator $\oplus$ ) of these three resistance values is about 5.5, which is less than the computed resistance 11.9. This reflects the fact that the three paths are not disjoint. The value 5.4 is computed under the implicit assumption that the three paths are disjoint, which is not the case here. Intuitively, having common exploits among different paths may increase the overall attack resistance, because the attacker must execute these exploits no matter what path they follow. Our metric naturally takes into consideration the overlapping portion of the paths. Above discussions also indicate that cumulative resistances can be computed in a breadth-first manner, which takes time $O\left(|\mathcal{E}|^{2}\right)$. 

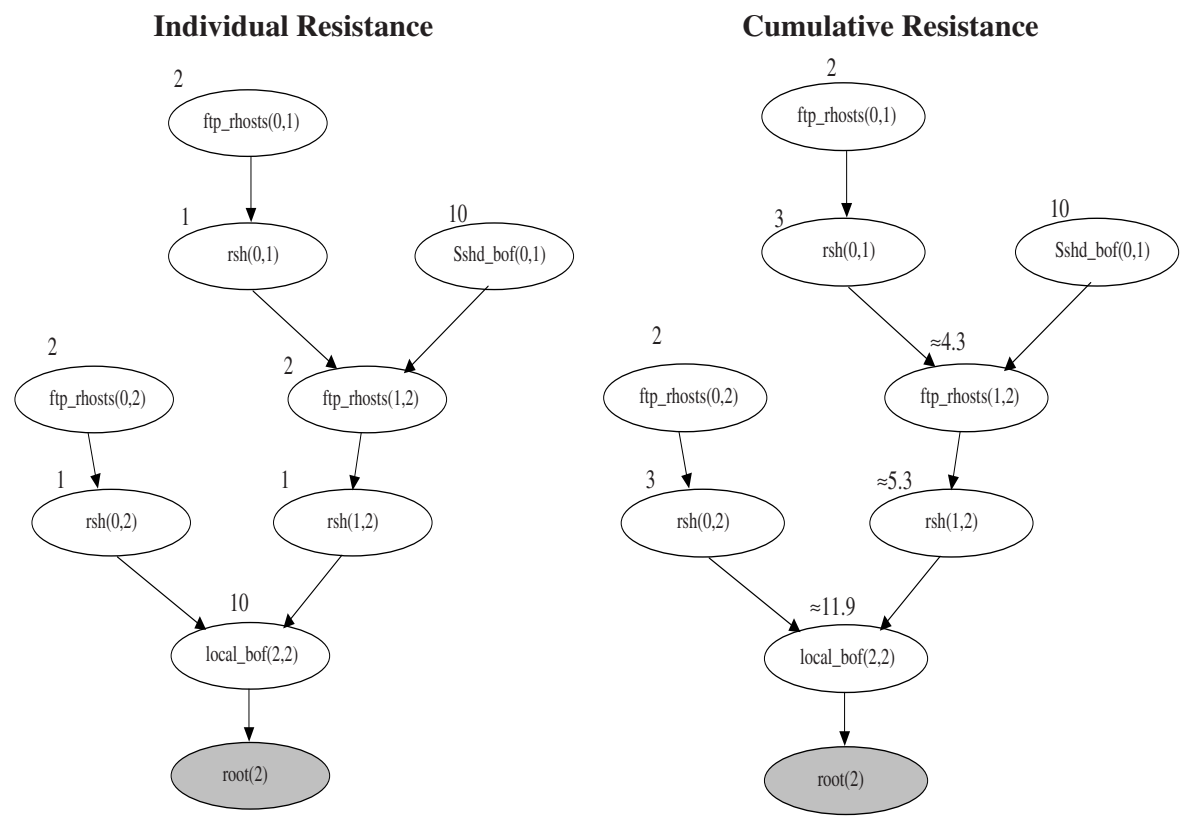

Fig. 2. An Example of Attack Resistance in Real Number

The function $\mathcal{R}$ Next we consider the function $\mathcal{R}$, that is the effect of executed exploits on the individual resistance of other exploits. The previous example is not sufficient for this purpose. Instead, we consider the abstract example given in the left hand side of Figure 3, where the dotted lines represent the following facts. Between exploit 1 and exploit 2, executing one will change the other's individual resistance from the original value $x$ to a new value $y$. Similar relationships exist between exploit 2 and exploit 3 , and between exploit 1 and exploit 6 . Notice the special notation between exploits 2, 5, and 7 , which denotes the conjunctive relationship between exploits 2 and 5. That is, exploit 7 cannot be executed unless both exploit 2 and 5 are already executed (this may happen when exploit 2 and exploit 5 both imply different conditions, and both conditions are required by the exploit 7 ).

The left hand side of Figure 3 shows three possibilities in dealing with the function $\mathcal{R}$. First, the effect of $\mathcal{R}(6)=(1, y)$ (that is, executing exploit 6 will change the individual resistance of exploit 1 as $r(1)=y$ ) can be safely ignored, because exploit 6 can never be executed before executing exploit 1 . On the other hand, the individual resistance $r(6)$ can now simply be changed from $x$ to $y$, because any execution of exploit 6 implies that exploit 1 has already been executed (which in turn implies a change in $r(6))$. Second, there is no naturally induced order between the execution of the exploit 1 and that of exploit 2, so they can be executed in any order. Intuitively, these two exploits meet at exploit 6 in the sense that we combine these two resistance values in the same formula when we compute $R(6)=1 /(1 /(r(1)+r(4))+1 / r(2))+r(6)$. At that point, the last composition operator used is $\oplus$ (that is, the exploit 4 and exploit 2 are 
Dependency Between Individual Resistances

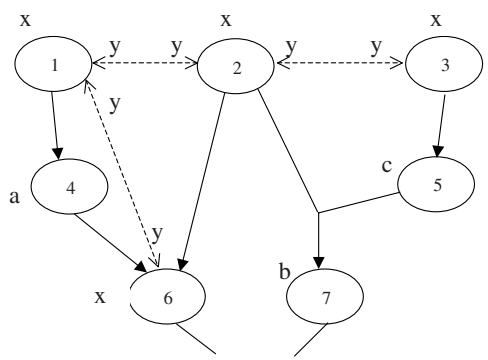

Dealing With the Non-Tree Structure

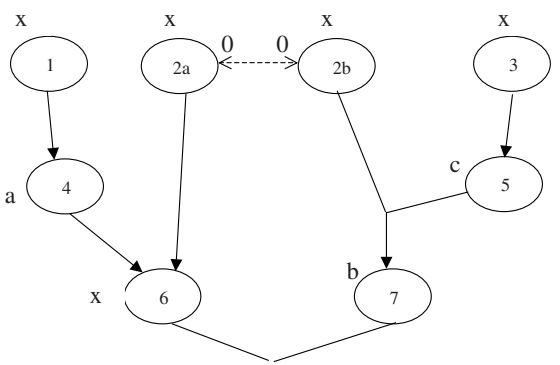

Fig. 3. Examples of the Function $\mathcal{R}$

disjunctive). We can then conclude that any minimal attack path (that is, an attack path with no proper subsets being a valid attack path) including exploit 6 will include either exploit 1 or exploit 2 , but not both. The effect of $\mathcal{R}(1)=(2, y)$ and $\mathcal{R}(2)=(1, y)$ can thus be ignored.

Third, when exploit 2 and exploit 3 meet at exploit 7 (when we compute $R(7)=$ $r(2)+r(3)+r(5)+r(7))$, the last composition operator we use is $\otimes$. This reflects the fact that the exploits 2 and 3 must both be executed in order to reach exploit 7 , although the executions can be in any order. If exploit 2 is executed before exploit 3 , then we have $r(2)=x$ and $r(3)=y$; if exploit 3 is first executed, we have $r(3)=x$ and $r(2)=y$. However, we can never have $r(2)=r(3)=y$ because a change only happens after an execution. In this case, we compute the cumulative resistance of exploit 7 for both cases: $r(2)=x, r(3)=y$ and $r(3)=x, r(2)=y$. We then choose the smaller result as the cumulative resistance of exploit 7 . This choice ensures that the computed cumulative resistance will be no greater than the cumulative resistance computed by following any attack path leading to exploit 7 . The above discussion covers all possible cases, because when two exploits eventually meet (that is, their resistances are combined), they must meet either at one of themselves (the case of the exploit 1 and exploit 6), or at a different exploit.

The Non-Tree Structure of Attack Graphs. Unlike the nice tree structure in the attack graph in Figure 1, it can be noticed that on the left hand side of Figure 3 both exploit 6 and exploit 7 depend on exploit 2, and the graph is not a tree. This is relevant because the cumulative resistance of this network should be different from another network where exploit 6 and exploit 7 depend on two different exploits. This issue, however, can be easily handled using the function $\mathcal{R}$ as follows. We split exploit 2 into two identical copies, say, exploit $2 \mathrm{a}$ and exploit $2 \mathrm{~b}$, as shown on the right hand side of Figure 3 . We then need the constraint that the resistance of these two exploits will never be added in computing a cumulative resistance, because they actually represent a single exploit. This constraint can be easily modeled as $\mathcal{R}(2 a)=(2 b, 0)$ and $\mathcal{R}(2 b)=(2 a, 0)$. We can now compute the metric as usual since the exploit dependency graph becomes a tree. 


\subsection{Attack Resistance as Sets of Initial Conditions}

We consider another concrete case where each exploit's individual attack resistance is the set of initial conditions (that is, conditions not implied by any exploit) required by that exploit. This measure can be easily obtained from the attack graph itself. The attack resistance in terms of the set of initial conditions has a very different meaning from the attack resistance discussed in the previous section. Here the resistance indicates conditions that must be satisfied before an intrusion is possible, instead of the effort and time spent during the actual intrusion. A weakest-adversary metric was recently proposed based on the set of initial conditions [21]. Different network configurations can be ordered based on their relative security, if a subset relationship exists between the sets of initial conditions required for reaching attack goals in the two attack graphs. We show that this metric is equivalent to a special case of our metric by using the set of initial conditions as individual resistance.
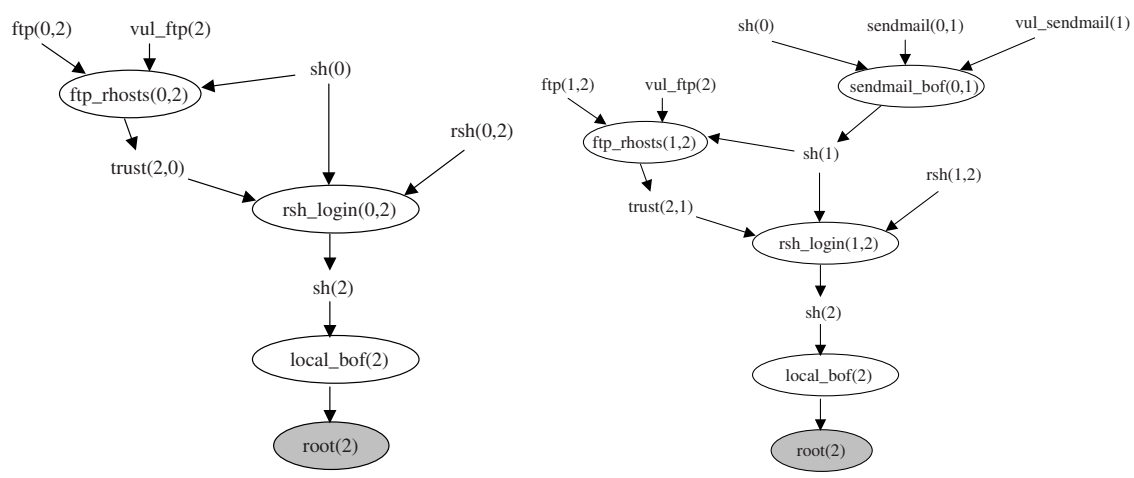

Fig. 4. Two Comparable Network Configurations

Figure 4/shows two network configurations that are comparable based on initial conditions [21]. The left hand side depicts an attack scenario similar to the one in Figure 1 but only involves two hosts. The right hand side shows a different scenario where the attacker is forced by a firewall to exploit the sendmail buffer overflow vulnerability on a third host as an intermediate step. It can be observed that in both cases the goal requires all the exploits to be executed, that is all the dependency relationship is conjunctive. We use set union as the operator $\otimes$ (we do not need the $\oplus$ operator in this case). The cumulative resistance of the exploit local_bof $(2)$ is thus simply the collection of all initial conditions in both cases. Clearly, the resistance in the first case is a proper subset of the resistance in the second case, and hence the second case has more resistance to potential attacks. This result is the same as reported previously [21].

\section{Related Work}

An overview of various issues relevant to security metric is recently given in the proceedings of the 2001 Workshop on Information Security System Scoring and Ranking 
[2]. The efforts by NIST on standardizing security metric are reflected in the Technology Assessment: Methods for Measuring the Level of Computer Security [18] and more recently in the Security Metric Guide for Information Technology Systems [27], which describes the current state of practice of security metrics, such as that required by the Federal Information Security Management Act (FISMA). Another overview of many aspects of network security metric is given in [10].

Closest to our work, Dacier et. al describe intuitive properties derived from common sense, which should be satisfied by any security metric [7/8 19]. They suggest to assess the difficulty of attacks in terms of time and effort spent by attackers. They assume an exponential distribution for an attacker's success rate over time. Based on this Markov model, they propose to use the MTTF (Mean Time to Failure) to measure the security of a network. They discuss simple cases of combining such measures but do not study the general case. We borrow some of the intuitive properties stated by them, but we use a different way for combining individual measures into the overall attack resistance and we consider a more general case represented by attack graphs.

Our approach of using additional functions for modeling the effect of executed exploits on the resistance value of other exploits is inspired by the work by Balzarotti et. al [3]. However, their work focuses on computing the minimum effort required for executing each exploit, whereas our work computes the overall security of a network with respect to given critical resources. Also, their work does not take into account the kind of dependency that we model using additional functions. Such dependency reduces the difficulty of executing an exploit while not directly enabling it to be exploitable. The work by Pamula et. al introduces a metric based on attack graph [21], in this paper we show that their metric is a special case of ours under certain conditions.

A qualitative measurement of the risk of a network is given based on various forms of the exploitability (that is, whether it is possible to compromise the network) [4]. Another series of work compares software for their relative vulnerabilities to attacks using a fixed set of dimensions, namely, attack surface [11|20|13]. The work by Mehta et. al borrows Google's PageRank methodology to rank exploits in an attack graph [14]. Their technique is especially suitable for threat models of worms or other malicious software that spread in a random way in a large network. Our metric has a different threat model, that is attackers have memory and are rational, so in most cases they will not follow a random model.

Metrics for other perspectives of security, especially trust in distributed systems, are relevant to our research. For example, Beth et. al proposed a metric for measuring the trust in an identity established through overlapping chains of certificates [5]. The way they combine values of trust in each certificate into an overall value of trust proves to be useful in our study. Similarly, the design principles given by Reiter et. al are intended for developing metric of trust, but we found these principles applicable to our study as well [24]. The formal logic language introduced for measuring risks in trust delegation in the RT framework inspires us to describe our metric using abstract operators [6].

To obtain attack graphs, topological vulnerability analysis evaluates potential multistep intrusions based on knowledge about vulnerabilities [7|9 19|22|33|28]. Such analyses can be either forward starting from the initial state [22[28] or backward from the goal state [25,26]. Model checking was first used to analyze whether a given goal state 
is reachable from the initial state [2325] and later used to enumerate all possible sequences of attacks between the two states [26]. To avoid the exponential explosion in the number of such explicit attack sequences, a more compact representation of attack graphs was proposed based on the monotonicity assumption saying an attacker never needs to relinquish any obtained capability [1]. On the attack response front, attack graphs have been used for the correlation of attacks, the hypotheses of alerts missed by IDSs, and the prediction of possible future attacks [2930].

\section{Conclusion}

Presently, qualitative and imprecise arguments are usually the basis for making decisions in securing a network. These arguments can mislead the decision making and as a result cause the reconfigured network to be in fact less secure. This paper described a novel attack graph-based attack resistance metric for measuring the relative security of network configurations. The main components of our metric are two composition operators for computing the cumulative attack resistance from given individual resistances. An additional function allowed the metric to take into consideration the dependency between individual attack resistances. We demonstrated the metric through two concrete cases. First, attack resistance was modeled as a real number, and the case was analogous to computing the resistance of a series-parallel circuit. We showed that the proposed metric satisfied intuitive requirements mentioned in the literature. Second, attack resistance was defined as the set of initial conditions required by each exploit. We showed that our metric in this case resembled the weakest-adversary metric previously proposed. It is our belief that the proposed metric will lead to novel quantitative approaches to vulnerability analysis, network hardening, and attack response.

Acknowledgements. This material is based upon work supported by National Institute of Standards and Technology Computer Security Division; by Homeland Security Advanced Research Projects Agency under the contract FA8750-05-C-0212 administered by the Air Force Research Laboratory/Rome; by Air Force Research Laboratory/Rome under the contract FA8750-06-C-0246; by Army Research Office under grant W911NF-05-1-0374; by Federal Aviation Administration under the contract DTFAWA-04-P-00278/0001; by National Science Foundation under grants CT-0627493, IIS-0242237, and IIS-0430402; and by Natural Sciences and Engineering Research Council of Canada under Discovery Grant. Any opinions, findings, and conclusions or recommendations expressed in this material are those of the authors and do not necessarily reflect the views of the sponsoring organizations. The authors are grateful to the anonymous reviewers for their valuable comments.

\section{References}

1. Ammann, P., Wijesekera, D., Kaushik, S.: Scalable, graph-based network vulnerability analysis. In: Proceedings of the 9th ACM Conference on Computer and Communications Security (CCS'02), pp. 217-224. ACM Press, New York (2002)

2. Applied Computer Security Associates. In: Workshop on Information Security System Scoring and Ranking (2001) 
3. Balzarotti, D., Monga, M., Sicari, S.: Assessing the risk of using vulnerable components. In: Proceedings of the 1st Workshop on Quality of Protection (2005)

4. Balzarotti, P., Monga, M., Sicari, S.: Assessing the risk of using vulnerable components. In: Proceedings of the 2nd ACM workshop on Quality of protection, ACM Press, New York (2005)

5. Beth, T., Borcherding, M., Klein, B.: Valuation of trust in open networks. In: Gollmann, D. (ed.) ESORICS 1994. LNCS, vol. 875. pp. 3-18. Springer, Heidelberg (1994)

6. Chapin, P., Skalka, C., Wang, X.S.: Risk assessment in distributed authorization. In: 3rd ACM Workshop on Formal Methods in Security Engineering: From Specifications to Code, ACM Press, New York (2005)

7. Dacier, M.: Towards quantitative evaluation of computer security. Ph.D. Thesis, Institut National Polytechnique de Toulouse (1994)

8. Dacier, M., Deswarte, Y., Kaaniche, M.: Quantitative assessment of operational security: Models and tools. Technical Report 96493 (1996)

9. Farmer, D., Spafford, E.H.: The COPS security checker system. In: USENIX Summer, pp. 165-170 (1990)

10. Hoo, K.S.: Metrics of network security. White Paper (2004)

11. Howard, M., Pincus, J., Wing, J.: Measuring relative attack surfaces. In: Workshop on Advanced Developments in Software and Systems Security (2003)

12. Jajodia, S., Noel, S., O'Berry, B.: Topological analysis of network attack vulnerability. In: Kumar, V., Srivastava, J., Lazarevic, A. (eds.) Managing Cyber Threats: Issues, Approaches and Challenges, Kluwer Academic Publishers, Dordrecht (2003)

13. Manadhata, K., Wing, J.M., Flynn, M.A., McQueen, M.A.: Measuring the attack surfaces of two ftp daemons. In: Quality of Protection Workshop (2006)

14. Mehta, V., Bartzis, C., Zhu, H., Clarke, E.M., Wing, J.M.: Ranking attack graphs. In: Recent Advances in Intrusion Detection (2006)

15. Noel, S., Jajodia, S.: Correlating intrusion events and building attack scenarios through attack graph distance. In: Yew, P.-C., Xue, J. (eds.) ACSAC 2004. LNCS, vol. 3189, Springer, Heidelberg (2004)

16. Noel, S., Jajodia, S., O’Berry, B., Jacobs, M.: Efficient minimum-cost network hardening via exploit dependency grpahs. In: Omondi, A.R., Sedukhin, S. (eds.) ACSAC 2003. LNCS, vol. 2823, Springer, Heidelberg (2003)

17. National Institute of Standards and Technology (Computer Security Division) (2007), http://nvd.nist.gov/

18. National Institute of Standards and Technology. Technology assessment: Methods for measuring the level of computer security. NIST Special Publication, pp. 500-133 (1985)

19. Ortalo, R., Deswarte, Y., Kaaniche, M.: Experimenting with quantitative evaluation tools for monitoring operational security. IEEE Trans. Software Eng. 25(5), 633-650 (1999)

20. Wing, J., Manadhata, P.: Measuring a system's attack surface. Technical Report CMU-CS04-102 (2004)

21. Pamula, J., Jajodia, S., Ammann, P., Swarup, V.: A weakest-adversary security metric for network configuration security analysis. In: Proceedings of the 2nd ACM workshop on Quality of protection, pp. 31-38. ACM Press, New York (2006)

22. Phillips, C., Swiler, L.: A graph-based system for network-vulnerability analysis. In: Proceedings of the New Security Paradigms Workshop (NSPW'98) (1998)

23. Ramakrishnan, C.R., Sekar, R.: Model-based analysis of configuration vulnerabilities. Journal of Computer Security 10(1/2), 189-209 (2002)

24. Reiter, M.K., Stubblebine, S.G.: Authentication metric analysis and design. ACM Transactions on Information and System Security 2(2), 138-158, 5 (1999) 
25. Ritchey, R., Ammann, P.: Using model checking to analyze network vulnerabilities. In: Proceedings of the 2000 IEEE Symposium on Research on Security and Privacy (S\&P'00), pp. 156-165. IEEE Computer Society Press, Los Alamitos (2000)

26. Sheyner, O., Haines, J., Jha, S., Lippmann, R., Wing, J.M.: Automated generation and analysis of attack graphs. In: Proceedings of the 2002 IEEE Symposium on Security and Privacy (S\&P'02), pp. 273-284. IEEE Computer Society Press, Los Alamitos (2002)

27. Swanson, M., Bartol, N., Sabato, J., Hash, J., Graffo, L.: Security metrics guide for information technology systems. NIST Special Publication, pp. 800-855 (2003)

28. Swiler, L., Phillips, C., Ellis, D., Chakerian, S.: Computer attack graph generation tool. In: Proceedings of the DARPA Information Survivability Conference \& Exposition II (DISCEX'01) (2001)

29. Wang, L., Liu, A., Jajodia, S.: An efficient and unified approach to correlating, hypothesizing, and predicting intrusion alerts. In: di Vimercati, S.d.C., Syverson, P.F., Gollmann, D. (eds.) ESORICS 2005. LNCS, vol. 3679, pp. 247-266. Springer, Heidelberg (2005)

30. Wang, L., Liu, A., Jajodia, S.: Using attack graphs for correlating, hypothesizing, and predicting intrusion alerts. Computer Communications 29(15), 2917-2933 (2006)

31. Wang, L., Noel, S., Jajodia, S.: Minimum-cost network hardening using attack graphs. Computer Communications 29(18), 3812-3824, 11 (2006)

32. Wang, L., Yao, C., Singhal, A., Jajodia, S.: Interactive analysis of attack graphs using relational queries. In: Proceedings of 20th IFIP WG 11.3 Working Conference on Data and Applications Security (DBSec 2006), pp. 119-132 (2006)

33. Zerkle, D., Levitt, K.: Netkuang - a multi-host configuration vulnerability checker. In: Proceedings of the 6th USENIX Unix Security Symposium (USENIX'96) (1996) 